\title{
TITLE:
}

\section{A p-adic analytic approach to the absolute Grothendieck conjecture}

$\operatorname{AUTHOR}(\mathrm{S})$ :

Murotani, Takahiro

\section{CITATION:}

Murotani, Takahiro. A p-adic analytic approach to the absolute Grothendieck conjecture. 代数幾何学シンポジウム記録 2018, 2018: 148-148

ISSUE DATE:

2018

URL:

http://hdl.handle.net/2433/236416

RIGHT: 


\section{A $p$-adic analytic approach to the absolute Grothendieck conjecture}

\section{Takahiro MUROTANI (RIMS, Kyoto University) Introduction}

Let $\boldsymbol{K}$ be a field of characteristic $0, \overline{\boldsymbol{K}}$ an algebraic closure of $\boldsymbol{K}, \boldsymbol{G}_{\boldsymbol{K}}$ the absolute Galois group of $\boldsymbol{K}, \boldsymbol{U}$ a hyperbolic curve over $\boldsymbol{K}$ and $\pi_{1}(U)$ the étale fundamental group of $\boldsymbol{U}$. We have the following natural exact sequence:

$$
1 \rightarrow \pi_{1}\left(U_{\bar{K}}\right) \rightarrow \pi_{1}(U) \stackrel{\text { pr }}{\rightarrow} G_{K} \rightarrow 1 .
$$

The relative and absolute Grothendieck conjectures ask:

$$
\begin{aligned}
& \text { relative : } \quad \pi_{1}(U) \rightarrow G_{K} \underset{\text { recoverable }}{\stackrel{?}{\rightarrow}} U, \\
& \text { absolute : } \quad \pi_{1}(U) \underset{\text { recoverable }}{\stackrel{?}{\rightarrow}} U \text {. }
\end{aligned}
$$

When $\boldsymbol{K}$ is a $\boldsymbol{p}$-adic field (i.e. a finite extension of $\mathbb{Q}_{p}$ ), the absolute version of Grothendieck conjecture (the absolute $\boldsymbol{p}$-adic Grothendieck conjecture) is unsolved. On the other hand, the absolute $\boldsymbol{p}$-adic Grothendieck conjecture is reduced to the problem of rational points of curves as follows:

Group-theoretic determination of whether or not $\boldsymbol{U}$ and its coverings admit rational points

$\frac{\begin{array}{c}\text { Group-theoretic characterization of } \\ \text { decomposition groups }\end{array}}{\Downarrow[\text { Mochizuki2] }}$

The absolute $\boldsymbol{p}$-adic Grothendieck conjecture

To consider this problem, we shall introduce the

" $\boldsymbol{i}$-invariant" of compact analytic manifold over a $\boldsymbol{p}$-adic field $\boldsymbol{K}$.

\section{Theorem-Definition [Serre]}

Let $\boldsymbol{q}$ be the cardinality of the residue field of $\boldsymbol{K}$ and $\boldsymbol{Y}$ a nonempty compact analytic manifold over $\boldsymbol{K}$. Then $\boldsymbol{Y}$ is the disjoint union of a finite number of balls and the number of (closed) balls is well determined $\bmod (q-1)$. We call the number of balls $i_{K}(\boldsymbol{Y}) \in \mathbb{Z} /(q-1) \mathbb{Z}$ the $\boldsymbol{i}$-invariant of $\boldsymbol{Y}$ over $\boldsymbol{K}$ Moreover, we set $i_{K}(\emptyset) \equiv 0 \bmod (q-1)$.

Let $\boldsymbol{X}$ be a proper hyperbolic curve over $\boldsymbol{K}$ and $\boldsymbol{X}(\boldsymbol{K})$ the set of $\boldsymbol{K}$-rational points of $\boldsymbol{X}$. In terms of the $\boldsymbol{i}$-invariants, the absolute $\boldsymbol{p}$-adic Grothendieck conjecture (for hyperbolic curves not necessarily proper) is reduced to the following two problems: e-mail: murotani@kurims.kyoto-u.ac.jp

\section{Question}

(A) Data of $\boldsymbol{i}$-invariants of sets of rational points of $\boldsymbol{X}$ and its étale coverings $\rightsquigarrow$ decomposition groups?

(B) Group-theoreticity of the $\boldsymbol{i}$-invariant of $\boldsymbol{X}(\boldsymbol{K})$ ?

Theorem $\mathrm{A}$ below gives a complete affirmative answer to $(A)$ and Theorem $B$ below gives a partial affirmative answer to $(B)$.

\section{Theorem A [M]}

Let $\boldsymbol{K}$ be a $\boldsymbol{p}$-adic field, $\boldsymbol{X}$ a proper hyperbolic curve over $\boldsymbol{K}$ and $\boldsymbol{q}$ the cardinality of the residue field of $\boldsymbol{K}$. Assume that $\boldsymbol{q} \neq \mathbf{2}$ and let $\boldsymbol{m}>\mathbf{1}$ be a divisor of

$q-1$. Then the following conditions are equivalent:

(i) $\boldsymbol{X}(\boldsymbol{K}) \neq \emptyset$.

(ii) $\exists \boldsymbol{X}^{\prime}$ : a finite étale covering of $\boldsymbol{X}$ such that $\boldsymbol{X}^{\prime}(\boldsymbol{K}) \neq \emptyset$.

(iii) $\exists \boldsymbol{X}^{\prime}$ : a finite étale covering of $\boldsymbol{X}$ such that $i_{K}\left(X^{\prime}(K)\right) \not \equiv 0 \bmod (q-1)$.

(iv) $\exists \boldsymbol{X}^{\prime}$ : a finite étale covering of $\boldsymbol{X}$ such that $i_{K}\left(X^{\prime}(K)\right) \not \equiv 0 \bmod \boldsymbol{m}$.

(v) $\exists \boldsymbol{X}^{\prime}$ : a finite étale covering of $\boldsymbol{X}$ such that $i_{K}\left(X^{\prime}(K)\right) \equiv($ a power of $p) \bmod (q-1)$.

\section{Theorem B [M]}

For $\boldsymbol{i}=\mathbf{1}, \mathbf{2}$, let $\boldsymbol{p}_{\boldsymbol{i}}$ be an odd prime number, $\boldsymbol{K}_{\boldsymbol{i}}$ a $\boldsymbol{p}_{\boldsymbol{i}}$-adic field and $\boldsymbol{X}_{\boldsymbol{i}}$ a proper hyperbolic curve over $\boldsymbol{K}_{\boldsymbol{i}}$ which has log smooth reduction (i.e. has stable reduction after tame base extension). Suppose that we are given an isomorphism of profinite groups $\alpha: \pi_{1}\left(X_{1}\right) \stackrel{\sim}{\rightarrow} \pi_{1}\left(X_{2}\right)$. Then we have

$$
i_{K_{1}}\left(X_{1}\left(K_{1}\right)\right) \equiv i_{K_{2}}\left(X_{2}\left(K_{2}\right)\right) \bmod 2 .
$$

In fact, by [Mochizuki1], if we are given an isomorphism $\alpha: \pi_{1}\left(X_{1}\right) \stackrel{\sim}{\rightarrow} \pi_{1}\left(X_{2}\right)$, we have $p_{1}=p_{2}$ (more precisely, we have $\boldsymbol{q}_{1}=\boldsymbol{q}_{2}$ where $\boldsymbol{q}_{i}$ is the cardinality of the residue field of $\boldsymbol{K}_{i}$ ). Moreover, $\boldsymbol{X}_{1}$ has log smooth reduction if and only if $\boldsymbol{X}_{2}$ has log smooth reduction.

\section{Remark}

If we prove Theorem B without assuming that $\boldsymbol{X}_{\boldsymbol{i}}$ has log smooth reduction, we can prove the absolute $\boldsymbol{p}$-adic Grothendieck conjecture for $\boldsymbol{p}$ odd.

[Mochizuki1] Shinichi Mochizuki, The absolute anabelian geometry of hyperbolic curves, Galois Theory and Modular Forms, Kluwer Academic Publishers, 2004, pp. 77-122. [Mochizuki2] Shinichi Mochizuki, Topics in absolute anabelian geometry II: Decomposition groups and endomorphisms, J. Math. Sci. Univ. Tokyo 20 (2013), pp. 171-269. [M] Takahiro Murotani, A p-adic Analytic Approach to the Absolute Grothendieck Conjecture, RIMS Preprint 1892 (August 2018).

[Serre] J.-P. Serre, Lie Algebras and Lie Groups, Lecture Notes in Mathematics 1500, Springer-Verlag, (1992).

[Tamagawa] Akio Tamagawa, The Grothendieck conjecture for affine curves, Compositio Mathematica 109 (1997), pp. 135-194. 